\title{
Photoisomeric molecular tagging velocimetry with CCVJ
}

\author{
Markus J. Schmidt $\odot,{ }^{*}$ Benno Käslin, and Thomas Rösgen $\odot$ \\ Institute of Fluid Dynamics, ETH Zurich, 8092 Zurich, Switzerland
}

(Received 4 September 2020; accepted 1 March 2021; published 6 April 2021)

\begin{abstract}
Various physical principles can be utilized for molecular tagging velocimetry (MTV). In this article, the capabilities and limitations of photoisomerization in MTV are studied. The molecular rotor 9-(2-carboxy-2-cyanovinyl)julolidine (CCVJ) exists in two isomers. Although the $E$ isomer, which is present in the absence of light, yields a fluorescent behavior, the $Z$ isomer is a photoproduct with no detectable luminescence. This regenerative behavior is utilized for MTV. The two time constants define the experimental limitations of the method for tagging and recovery. It is shown that, whereas the tagging time constant is in the range of milliseconds to seconds, the recovery time is in the range of minutes to hours, enabling measurements of slow flow phenomena. A flow in a rectangular channel with a Reynolds number of $9 \times 10^{-3}$ and a Péclet number of 1009 is investigated. Furthermore, the addition of methyl- $\beta$-cyclodextrin (methyl- $\beta$-CD) to the solution increases the fluorescent quantum yield and the isomeric rate constants, supporting the hypothesis of a complex formation with CCVJ. It is shown that photoisomeric MTV is suitable for analyzing slow flow regimes in the liquid phase. The addition of methyl- $\beta$-CD increases the dynamic range of the results. In comparison with existing MTV principles, photoisomerization has the advantage of an intrinsic regenerative behavior. The cost-efficient and simple setup make it an alternative in biological and environmental flow studies.
\end{abstract}

DOI: 10.1103/PhysRevFluids.6.044101

\section{INTRODUCTION}

In the field of molecular tagging velocimetry (MTV), several photochemical phenomena have been utilized to measure fluid velocities [1,2]. The seeding with photochromic dyes is suitable for measurements of flows, but the illumination scheme produces low contrast images, whereas the advantage is the ability of regeneration. For example, caged dyes can exhibit a high signal-to-noise ratio and be interrogated a long time after the photolysis, but the conversion is permanent $[3,4]$. In contrast, phosphorescent supramolecules can be excited for use in MTV, but measurements are limited by the lifetime of the dye [5].

One possibility for imaging molecules in a liquid flow is the photoactivated nonintrusive tracking of molecular motion. Lempert et al. [3] were among the first to apply it to a fluid flow. In their work, photocaged molecules are first tagged with a "write" laser, activating the dye's fluorescence. Afterward, a "read" laser is used for the tracking of these molecules. The use of caged dyes is by now a well-established method [3,4,6-10]. In addition, photobleaching has been successfully applied to MTV. Herein the continuous illumination of the dye reduces its ability to fluoresce.

\footnotetext{
*schmmark@ethz.ch
}

Published by the American Physical Society under the terms of the Creative Commons Attribution 4.0 International license. Further distribution of this work must maintain attribution to the author(s) and the published article's title, journal citation, and DOI. 
However, for effective use of this experimental fluid dynamics method, the photon flux has to be high enough to achieve distinguishable intensity levels. Since the photon flux usually leads to photochemical oxidation, the process is irreversible. Various experiments have demonstrated the use of photobleaching in MTV [11-16].

Molecular tagging velocimetry is not only applied to liquids but to gases as well. For highly turbulent flows, several methods are available. Among those methods are direct excitation of biacetyl [17] and hydroxyl [18], krypton tagging velocimetry [19-22], femtosecond laser electronic excitation tagging velocimetry [23-26], and vibrational excited NO monitoring [27-29].

In this paper, we present the capabilities and limitations of photoisomerization as a method for tagging and tracking ensembles of molecules in a liquid. In chemistry, photoisomers are molecules with identical composition but different structures, whereas the current concentration of the isomers depends on the photon flux density. Two requirements must be fulfilled for the utilization of photoisomerization. First, the quantum efficiencies of fluorescence must differ. Ideally, one isomer should not fluoresce at all. Second, the isomerization rate constants must be suitable for tagging and tracking in a flow. Consequently, one of the rate constants should be in the range of milliseconds for tagging whereas the other in the range of minutes to hours for a successful acquisition of the tag.

Photoisomerization in MTV was introduced by Namykin and co-workers [30,31]. The dye Evans Blue exhibits fluorescence in complexes with blood plasma proteins. The existence of two isomers is proposed with the tagged trans-isomer exhibiting a higher fluorescent quantum yield and a useful rate-defined time window for measurements of $1 \mathrm{~s}$, which limits the possible range of applications.

Recently, the fluorescent behavior of the dye and molecular rotor 9-(2-carboxy-2cyanovinyl)julolidine (CCVJ) in fluid flow has been proposed for different diagnostic applications. One scenario relied on an assumed shear dependency of the dye [32-34], whereas a subsequent study indicated that the photoisomeric behavior of CCVJ might be responsible for the observed changes in fluorescence [35]. This interpretation was confirmed in further experiments [35,36]. The deployment of cyclodextrins together with CCVJ might lead to an increase in the fluorescence [37]. Independent of Namykin and co-workers [30,31], CCVJ has been utilized for photoisomeric MTV by the authors [38].

In this paper, we experimentally examine the relevant photochemical properties of CCVJ for application in photoisomeric MTV. Furthermore, an application to a canonical flow, the Poiseuille flow in a rectangular channel, is presented, with the measurement time reaching the limit due to diffusion of the dye [4].

\section{METHOD}

\section{A. Photoisomerization kinetics}

CCVJ exists in two isomers. Next to differences in the chemical structure, one significant difference is the quantum yield for luminescence with luminescent efficiencies significantly higher for the $E$ isomer. This property of CCVJ and other dyes can be utilized for MTV.

The conversion of the $E$ isomer to the $Z$ isomer is driven by absorption of photons, whereas the backreaction is thermal. It explains the difference in timescales as shown in the results for the time constants in Secs. IV B and IV C. Although the photoproduct rate constant is in the range of milliseconds to minutes, the backreaction can take place on the order over tens of minutes.

Rumble et al. [35] have demonstrated that the luminescent $E$ isomer is dominant in the dark at room temperature, whereas the $Z$ isomer is present as well at constant illumination.

The dynamics of isomerization are a complex phenomenon, which is described in detail by Satiel and Sun [39]. For the application of the effect in flow diagnostics, the characteristic time constants for the two isomerization processes are of significance. From this point of view, the simplified state of the system regarding the concentration of the $E$ isomer can be described by

$$
\frac{\partial c_{E}(t)}{\partial t}=-k_{E Z}\left[\left(c_{E}(t)-c_{E, \min }\right]+k_{Z E}\left[c_{E, \min }+\Delta c_{E}-c_{E}(t)\right]\right.
$$


with $c_{E}(t)$ as the actual concentration of the $E$ isomer, $c_{E \text {, min }}$ as the minimal concentration in brightadapted solution, $\Delta c_{E}$ as the difference in concentration between the dark- and the bright-adapted solutions, and $k_{i j}$ as the reaction rate constant from the $i$ to $j$ isomer [39]. The use of $c_{E \text {, min } \text { and }}$ $\Delta c_{E}$ is introduced based on the report of Rumble et al. [35] describing that the isomerization is not only complete, but only converts a fraction of the $E$ isomer. The rate constant $k_{E Z}$ depends on the photon flux present in the reaction, whereas $k_{Z E}$ does not exhibit a photosensitive behavior and is exclusively thermally driven. A general solution of Eq. (1a) is given by

$$
c_{e}(t)=C \exp \left[-\left(k_{E Z}+k_{Z E}\right) t\right]+\frac{\left(k_{E Z}+k_{Z E}\right) c_{E, \min }+k_{Z E} \Delta c_{E}}{k_{E Z}+k_{Z E}}
$$

with $C$ as an integration constant.

From the perspective of experimental fluid dynamics, it is more instructive to think in terms of time constants than in rate constants. Thus, tagging time constants are introduced to represent the isomerization dynamics of CCVJ. One process of isomerization is due to illumination with light, and, hence, the relation with the rate constant from the $E$ to the $Z$ isomers is given by $\tau_{\text {tag }}=k_{E Z}{ }^{-1}$, defined as the tagging time constant. Similarly, the recovery time constant is defined, representing the characteristic timescale for the backisomerization from the $Z$ to the $E$ isomer. The relation to the corresponding rate constant is given by $\tau_{\text {rec }}=k_{Z E}{ }^{-1}$.

Two particular solutions are of interest from an experimental point of view and applied for the determination of the tagging time constants. First, a dark-adapted solution is considered with a concentration of the $E$ isomer of $c_{E \text {, min }}+\Delta c_{E}$. This solution is then illuminated with a laser, leading to a nonzero isomerization rate $k_{E Z}$. Furthermore, it is assumed that the thermal backisomerization is much slower $k_{Z E} \ll k_{E Z}$. In addition, the initial condition $c_{E \text {, tag }}(0)=c_{E \text {, min }}+\Delta c_{E}$ is used for the computation of the integration constant. Equation (1b) then simplifies to

$$
c_{E, \mathrm{tag}}(t)=\Delta c_{E} \exp \left(-t / \tau_{\mathrm{tag}}\right)+c_{E, \min },
$$

with $\tau_{\text {tag }}=k_{E Z}{ }^{-1}$ as the tagging time constant.

Second, after a sufficiently long illumination time, the concentration of the $E$ isomer reaches its minimum, which is called the light-adapted solution. If the illumination is stopped in this case, the concentration change solely depends on the thermal reaction with no photoisomerization rate $k_{E Z}=0$. The initial condition is given by $c_{E \text {,rec }}(0)=c_{E \text {,min }}$. This leads to the solution,

$$
c_{E, \text { rec }}(t)=\Delta c_{E}\left[1-\exp \left(-t / \tau_{\text {rec }}\right)\right]+c_{E, \min },
$$

with $\tau_{\text {rec }}=k_{Z E}{ }^{-1}$ as the recovery time constant. The time constants can be determined in experiments. It has to be emphasized that the tagging time constant $\tau_{\text {tag }}$ depends on the photon flux produced the light source used for the tagging and, thus, is only constant with constant illumination, whereas the recovery constant $\tau_{\text {rec }}$ depends on the temperature.

The derived relations are applied for the experimental determination of the recovery and tagging time constants. Although the tagging time constant provides a measure of the laser's required power density concerning the achievable intensity contrast and the achievable timescales, the recovery time constant is determined to define a measure for the achievable contrast during long-term experiments.

\section{B. Complex formation with cyclodextrin}

An increase in fluorescence has been reported for CCVJ in the presence of methyl- $\beta$ cyclodextrin (methyl- $\beta$-CD) [37]. This suggests the formation of supramolecules similar to those used in MTV with direct phosphorescence [5].

By their chemical structure, cyclodextrins form a nanocavity from their aromatic rings. $\alpha, \beta$, and $\gamma$ cyclodextrins are species with increasing sizes of their nanocavities. They were examined regarding their influence on the fluorescence quantum yield and lifetime. With increasing nanocavity size and molar concentration, the addition of cyclodextrins to CCVJ solutions increases both the quantum yield and fluorescence lifetime of the dye [37]. 
The results indicate a complex formation of cyclodextrins with CCVJ and an influence on the molecular rotation of the photoisomeric dye. This capability suggests a potential use for tagging velocimetry by increasing the quantum yield and modifying the reaction rate constants.

\section{Photoisomeric tagging}

Photoisomeric MTV is simple in application and cost efficient due to the regeneration of the dye, i.e., the slow reconversion from the $Z$ to the $E$ isomer in darkness. With suitably high rate constants of the isomerization process, it is possible to extract velocity information in flow regimes.

In general, the $E$ isomer solution is tagged with a suitable laser line to produce a local increase in the nonfluorescent $Z$ isomer. The solution can be illuminated entirely afterward, and the tagged region with the $Z$ isomer yields a lower fluorescence signal than the rest of the solution.

The effect of velocimetric tagging using photoisomerism is comparable to that of photobleaching of molecules with the resulting images being inverted; the tagged region exhibits a lower level of intensity. Advantages can be found in the regenerative behavior of the dye. Compared to photochromic dyes, CCVJ has the advantage of being soluble in water and other organic solvents and allowing lower-powered lasers since no tautomers have to be created.

\section{Flow regime}

A Poiseuille flow in a rectangular duct is chosen as a slow flow scenario to demonstrate the capabilities and limitations of photoisomeric MTV. The examined flow is characterized by the Reynolds number $\operatorname{Re}=\frac{u d_{h} \rho}{\mu}$ with $u$ as the average velocity in the channel, $d_{h}$ as the hydraulic diameter, and $\rho$ and $\mu$ as the density and dynamic viscosity of the fluid, respectively. In the presented experiments, $d_{h}$ is computed as $3.75 \mathrm{~mm}$, and the fluid properties are represented by ethylene glycol at standard conditions $\left(\rho=1.1132 \mathrm{~g} / \mathrm{cm}^{3}, \mu=1.61 \times 10^{-2} \mathrm{~N} \mathrm{~s} \mathrm{~m}^{-2}\right)$. The velocity $u$ is derived from the known piston speed of the syringe pump.

Since the observed timescales can be within the order of magnitude for molecular diffusion, the Péclet number as a ratio of advective and diffusional transport rates is computed to examine the influence of diffusion. It is defined as $\mathrm{Pe}=u d_{h} D^{-1}$ with $D$ as the mass diffusion coefficient.

\section{EXPERIMENTAL SETUP}

Figure 1 shows the schematic setup. A JAI RM-4200GM camera with a Nikon AF NIKKOR 55-mm f/3.5 lens is used for acquisition. A Nikon PK-12 14-mm distance ring provides additional magnification in the small region of interest. The camera is equipped with a Schott OG 515 highpass filter with a nominal cutoff wavelength of $515 \mathrm{~nm}$. For tagging of the fluid, an RLDE405M100-5 diode laser from Roithner LaserTechnik GmbH with a wavelength of $405 \mathrm{~nm}$ and a power of $100 \mathrm{~mW}$ is used. The initial diameter of the laser beam of $5 \mathrm{~mm}$ is focused with an optical lens. For the final illumination and image acquisition, a custom-made light-emitting diode (LED) cluster is applied. The LED cluster consists of 61 single LEDs with a nominal power of $120 \mathrm{~mW}$ each. Each LED is a Sloan L5-B91G-WT with a nominal wavelength of $470 \mathrm{~nm}$. The LED cluster is actively cooled to increase its durability. The light sources and the camera are controlled with a Berkeley Nucleonics Model 500A delay generator. The exposure time is controlled with the delay generator. The fluid flow is established with a Cavro XLP 6000 modular syringe pump with a 5-ml syringe.

A solution of $15-\mu \mathrm{mol} \mathrm{L}^{-1} \mathrm{CCVJ}$ in ethylene glycol is used. For the experiment with methyl- $\beta$ $\mathrm{CD}$, the solution is supplemented with the cyclodextrin at a concentration of $3 \mathrm{mmol} \mathrm{L}^{-1}$. The fluid is pumped through a flow channel with $5-\mathrm{mm}$ width, 3-mm depth, and 100-mm length. The flow chamber is made of acrylic glass. A black plastic sheet is placed on the bottom of the channel to block light from the background.

For a typical acquisition, the pump is started to establish the flow. Then an acquisition with an activated LED is taken of the constant flow in the chamber to measure a reference fluorescence signal without tagging. Next, the laser is triggered to tag a thin line in the flow. The excited CCVJ 


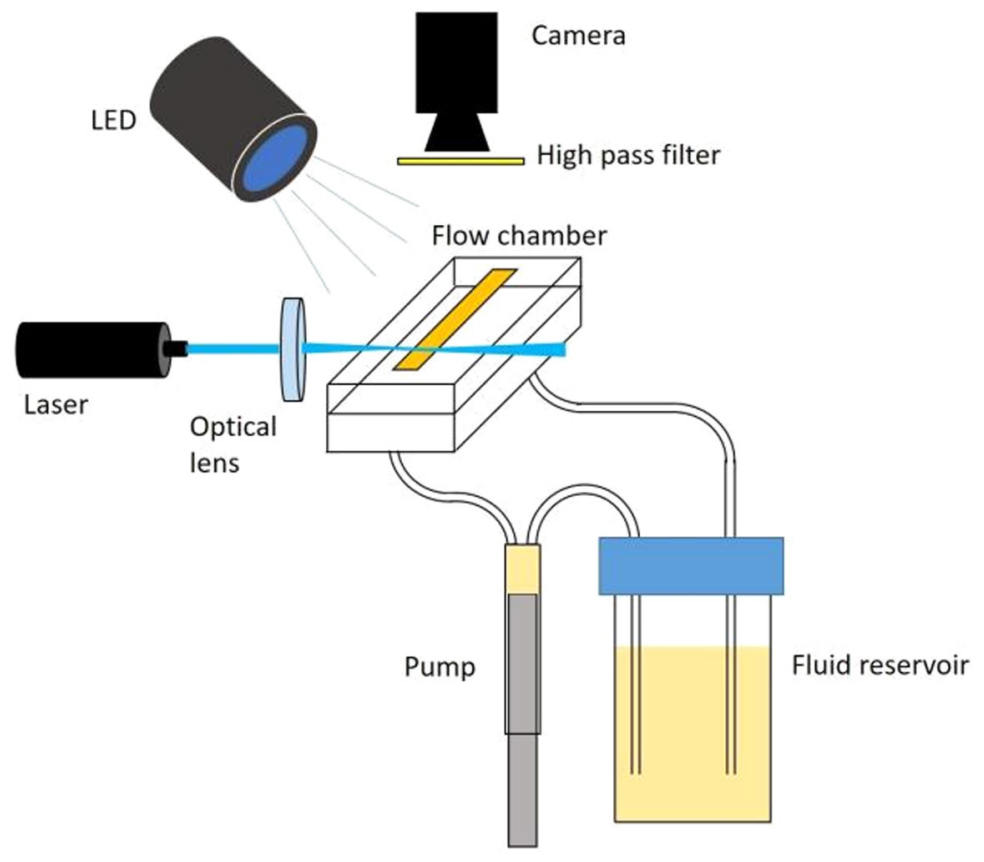

FIG. 1. Experimental setup.

molecules change to the $Z$ isomer and lose their fluorescent property. Finally, the fluorescence of the CCVJ molecules in the chamber is stimulated with the LED lamp and captured. The experiment is repeated several times to increase the signal-to-noise ratio of the results.

\section{RESULTS}

\section{A. Diffusion coefficient}

For the determination of the diffusion coefficient $D$ of CCVJ in ethylene glycol, the flow chamber is replaced with a cuvette. The laser beam is expanded to illuminate the cuvette's complete width and cutoff with a knife edge, resulting in a planar tagging layer. Three independent experiments were conducted with diffusion times of 100, 200, and 250 s between tagging and image acquisition to study the evolution of the diffusion front. Four images are taken for each diffusion time with a pause of $7200 \mathrm{~s}$ for the solution to adapt to the initial state again. These images are background corrected and averaged. For the computation of the diffusion coefficient, this mean image is background subtracted and normalized with a no-tag image, only illuminated with the LED. The relative intensities are averaged in the direction of the laser beam propagation. The resulting data points can be found in Fig. 2 as data.

Under the assumption of a one-dimensional diffusion for the tagging front, the solution of Fick's second law becomes

$$
I(x, t)=I_{0} \operatorname{erfc}\left(\frac{x-x_{\text {off }}}{2 \sqrt{D t}}\right)+I_{\text {off }},
$$

with $D$ as the diffusion coefficient, $x$ as the coordinate perpendicular to the laser beam propagation, $x_{\text {off }}$ as the respective offset, $I_{0}$ as half the relative intensity between the tag and the ambient, and $I_{\text {off }}$ as a relative intensity offset. The tagged cuvette has basically two observable diffusion fronts. The visually sharper upper front is used for analysis. Since the cuvette is only partially illuminated in the observation direction, the contrast is significantly lower than in the 
(a) $100 \mathrm{~s}$

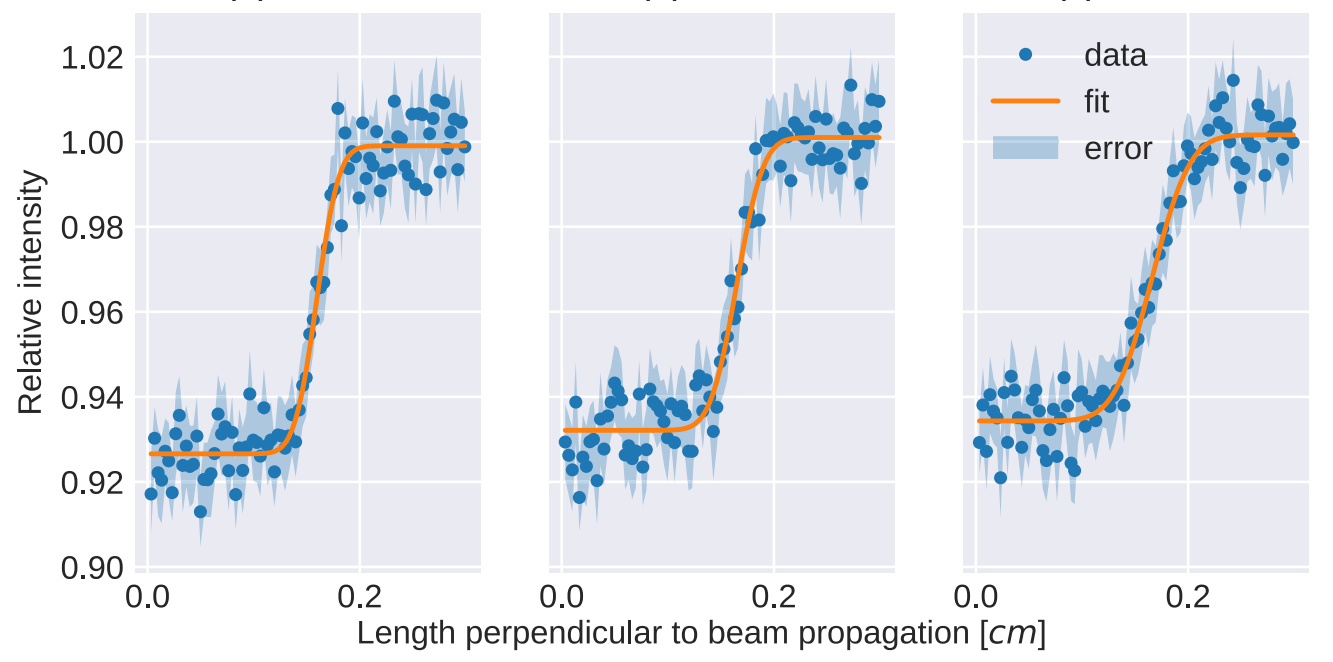

FIG. 2. Diffusion of a tagging front in a cuvette after 100, 200, and $250 \mathrm{~s}$. The absolute intensity values are normalized with a reference image without tag and averaged in the beam direction. The blue dots denote the data with their respective sample standard errors whereas the line represents a fit of Eq. (4).

other experiments. The model is fitted to the data with the PYTHON package LMFIT in version 0.9.9 [40]. The results can be found in Fig. 2. The fitted values for the diffusion coefficient are $D=(1.35 \pm 0.31) \times 10-6 \mathrm{~cm}^{2} \mathrm{~s}^{-1}(100 \mathrm{~s}), D=(0.95 \pm 0.21) \times 10^{-6} \mathrm{~cm}^{2} \mathrm{~s}^{-1}(150 \mathrm{~s})$, and $D=(1.56 \pm 0.28) \times 10^{-6} \mathrm{~cm}^{2} \mathrm{~s}^{-1}(250 \mathrm{~s})$, which is on the same order of magnitude as the selfdiffusivity of ethylene glycol with $0.961 \times 10^{-6} \mathrm{~cm}^{2} \mathrm{~s}^{-1}$ [41]. For the computation of the Péclet number the mean value of $1.29 \times 10^{-6} \mathrm{~cm}^{2} \mathrm{~s}^{-1}$ is used. Data are available in a repository [42].

\section{B. Recovery time constant}

One advantage of the use of photoisomers in MTV is the backisomerization after tagging. For this recovery, the rate of change from the $Z$ to the $E$ isomer is relevant, characterized by the recovery time constant $\tau_{\text {rec }}$.

The setup is identical to the measurement of the diffusion coefficient in the preceding paragraph. The experiments are conducted at room temperature. The cuvette is illuminated with the LED for $60 \mathrm{~s}$ to establish a complete conversion in favor of the $Z$ isomer. Subsequently, the probe is illuminated for $0.1 \mathrm{~s}$ by the LED for image acquisition. After one acquisition, the sample is tagged entirely again, and the delay between tagging and acquisition is increased. The exposure time is set short compared to the tagging time constant. Thus, no influence on the recovery is assumed. The data are readily available in a repository [42].

The data are fitted with LMFIT to Eq. (3). The resulting recovery time constant is $\tau_{\text {rec }}=(1050 \pm$ 131) s and a goodness of fit of $\chi^{2}=3.48 \times 10^{-4}$. With the results of the tagging time constant in next paragraph, it will become apparent that the recovery time is significantly longer than the tagging time constant, enabling measurements of long-lived phenomena (see Fig. 3).

An optimization result with a double-exponential has been computed. It is not shown in this article since the optimization did not identify a second lifetime.

\section{Tagging time constant}

The tagging time is a measure for the effective intensity difference, which can be achieved with the method, depending on the laser power density. On the one hand, the power density or, for a 


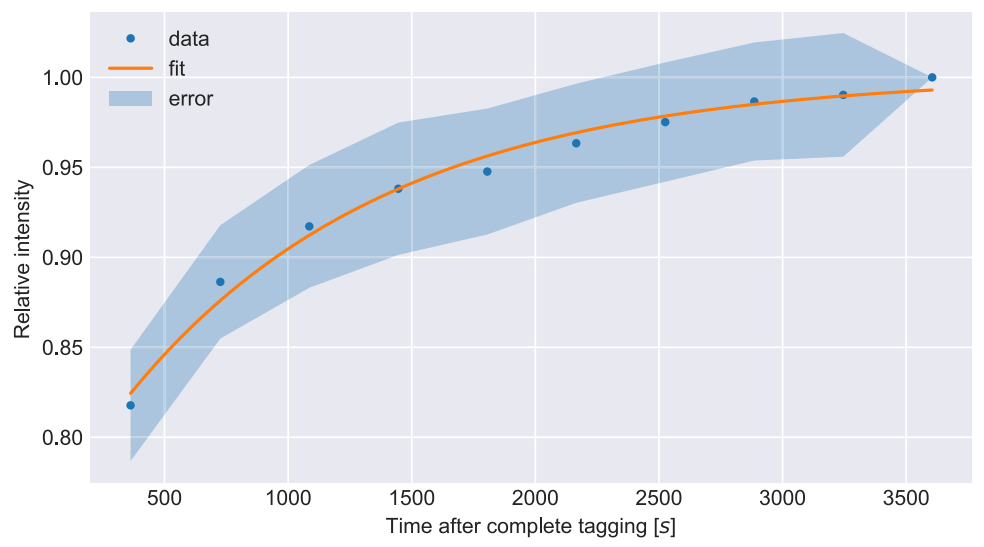

FIG. 3. Determination of the recovery time constant after complete tagging of the cuvette. Data represent the average of three experiments and are normalized with the last acquired data point. The results of the fit lie within the standard error.

pulsed laser, energy density has to be sufficiently high to realize a measurable energy contrast. On the other hand, for lower power lasers, such as the one used in the presented experiments, the tagging time may be too long concerning the flow velocities, effectively smearing the tag.

The flow cell is replaced with a cuvette to determine the influence of laser power on the tagging time constant $\tau_{\text {tag. }}$. The sample is placed again in darkness to achieve a dark-adapted solution. The solution is then tagged for a specified time with the laser and an image with LED illumination taken after $1 \mathrm{~ms}$. For the subsequent acquisition, the tagging time is increased, and a pause of $7200 \mathrm{~s}$ is introduced to establish a dark-adapted solution again. The laser power density variation is established with a set of neutral-density (ND) filters, which are placed between the laser and the cuvette. For each laser power density setting, five measurements are averaged, and the tagging time constant is derived from a fit of Eq. (2) with LMFIT to the data. The averaged data and the fits of can be found in Fig. 4, and detailed results of the fits can be found in Table I. The effective laser power

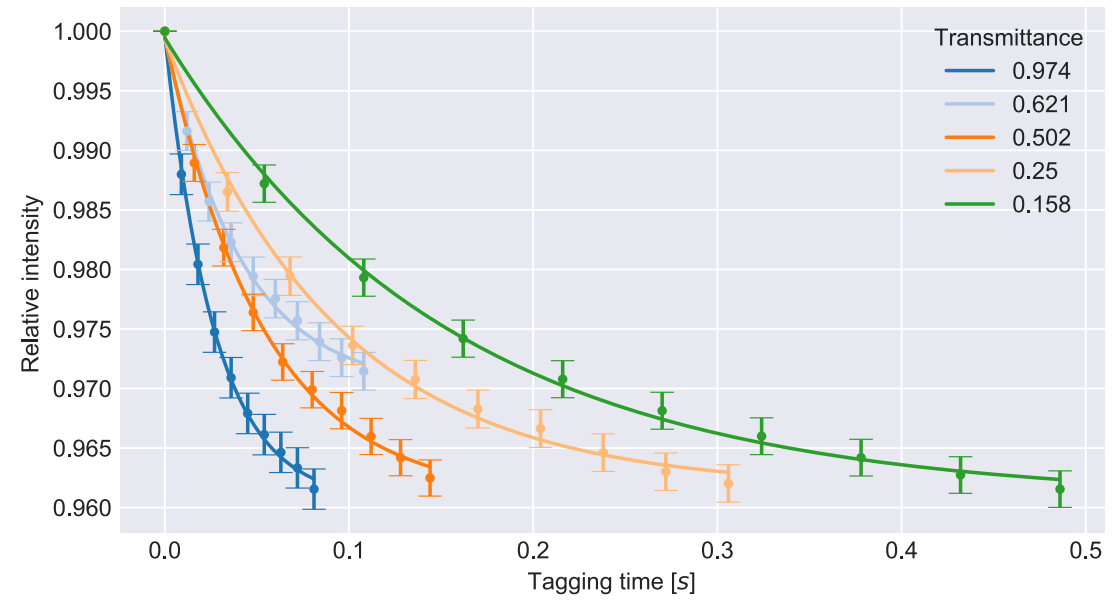

FIG. 4. Relative intensity decay data (circles) and standard error (bars) for different transmittances of the ND filters. The solid lines represent the respective fits. Note that the measurement with transmittance 0.621 is acquired with a different tagging volume, resulting in a different relative intensity decay. 
TABLE I. Tagging time constant in dependence on laser power density.

\begin{tabular}{lccccc}
\hline \hline Power density $\left(\mathrm{W} / \mathrm{cm}^{2}\right)$ & 0.50 & 0.32 & 0.26 & 0.13 & 0.08 \\
\hline$\tau_{\text {tag }}(\mathrm{ms})$ & 26.8 & 41.2 & 53.2 & 91.7 & 154.1 \\
$\tau_{\text {tag }}$ confidence interval $1 \sigma(\mathrm{ms})$ & 1.0 & 2.9 & 3.0 & 6.0 & 8.3 \\
Amplitude $\left(10^{-2}\right)$ & 3.92 & 2.96 & 3.88 & 3.77 & 3.87 \\
Amplitude error $\left(10^{-2}\right)$ & 0.05 & 0.07 & 0.08 & 0.09 & 0.07 \\
$\chi^{2}\left(10^{-6}\right)$ & 1.51 & 2.39 & 2.79 & 4.90 & 3.19 \\
\hline \hline
\end{tabular}

density is computed from the nominal power of $100 \mathrm{~mW}$ and the used ND filters. The measurement data can be found in a separate data repository [42].

Note that the measurements are not suitable for determining the absolute intensity reduction due to the chosen tagging volume. As an illustrative example, the laser beam has been cropped for the acquisition with a relative transmittance of 0.621 . Since the laser only partially illuminates the cuvette, but the LED produces global illumination, the resulting signal is a mix of tagged and untagged luminescence signals. The difference in amplitudes can be found in Table I. Consequently, the amplitude, defined as the relative intensity difference between the isomers, differs from the other measurements. Figure 5 shows a decrease in tagging time for increasing laser power.

The data have been fitted with a double-exponential without finding a second lifetime.

Since the tagging time constant is dependent on the photon flux density, it is instructive to relate the laser power density as a measure of the photon flux density to the achieved tagging time. Results can be found in Fig. 5.

\section{Measurement of a Poiseuille flow}

For sharp tags, the laser activation time has to be kept as short as possible. Since the recovery time constant $\tau_{\text {rec }}$ is on the same order of magnitude as the observation time, the tagging has to be long enough to achieve a sufficient signal-to-noise ratio.

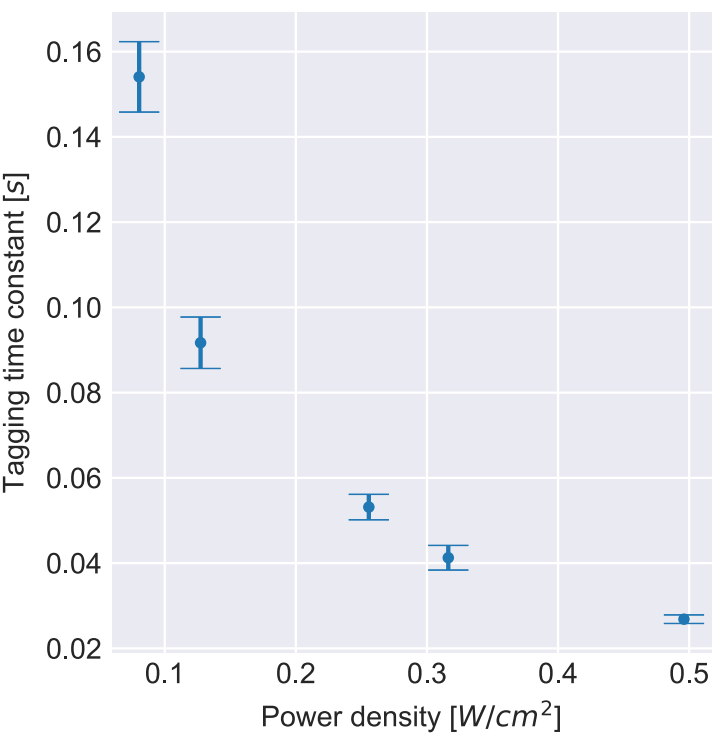

FIG. 5. Dependency of the tagging time constant on laser power density and the respective $1 \sigma$ confidence interval. 


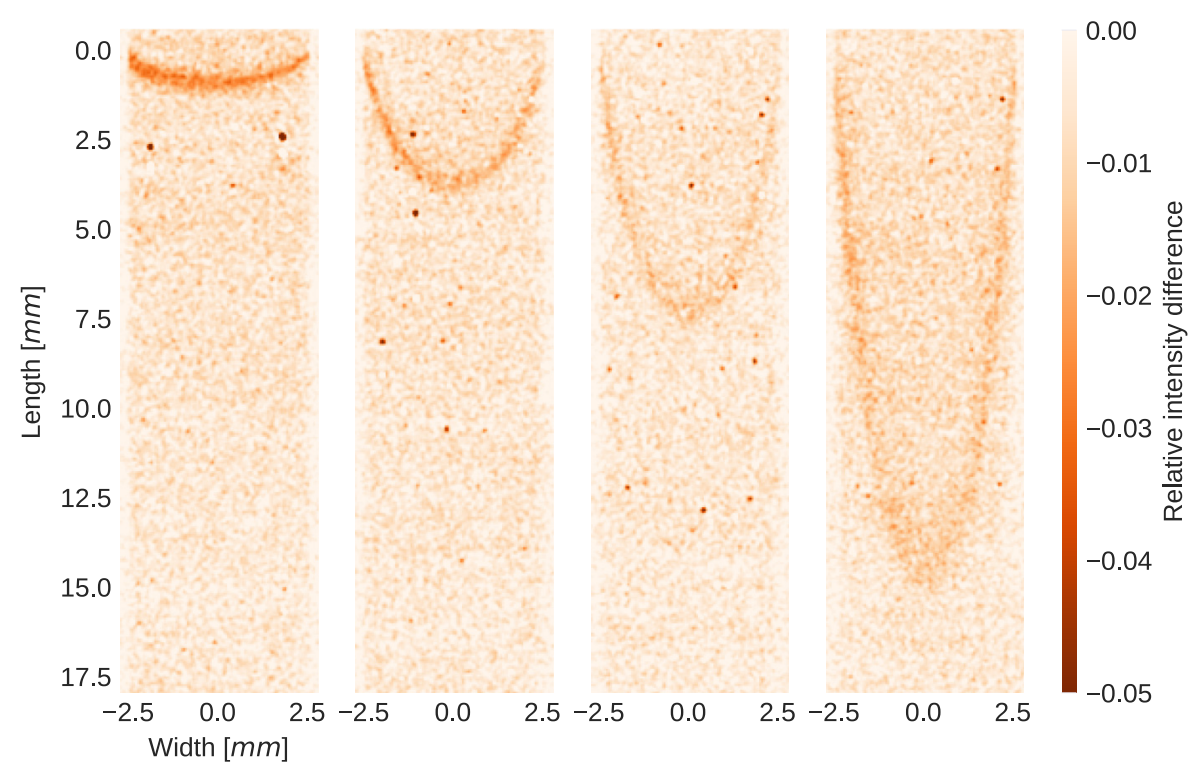

FIG. 6. Relative intensity difference with $\mathrm{Re}=9 \times 10^{-3}$ and $\mathrm{Pe}=1009$ at $10,50,100$, and $200 \mathrm{~s}$ (left to right) after a tag of $0.05 \mathrm{~s}$ for a single acquisition. The standard deviation of the relative signal is 0.0047 .

Within a limit of $200 \mathrm{~s}$, it has been found that a suitable tagging time of the laser is $0.05 \mathrm{~s}$ to achieve a sufficient contrast for the experiments.

The average speed $u$ during the experiment is $0.0347 \mathrm{~mm}$ as computed by the stroke speed of the syringe pump. This results in $\operatorname{Re}=9 \times 10^{-3}$ and $\mathrm{Pe}=1009$. The gain of the camera is set to $25 \%$ and the $\mathrm{f} / \#$ of the objective to 8 . The exposure time of the camera and the activation of the LED cluster is $0.8 \mathrm{~s}$. Only one acquisition is taken after each isomerization tag of the laser to increase the contrast of the results.

The results are shown as relative intensity difference $I_{\text {rel }}$. The relative intensity difference is defined as the difference of the tagged flow image $I_{\text {tag }}$ and a reference image without a tag $I_{\text {ref }}$ per pixel, normalized by the global mean of the reference image $\bar{I}_{\text {ref }}$,

$$
I_{\text {rel }}(x, y)=\frac{I_{\text {tag }}(x, y)-I_{\text {ref }}(x, y)}{\bar{I}_{\text {ref }}} .
$$

Figure 6 shows results for 10, 50, 100, and $200 \mathrm{~s}$ after tagging for a single acquisition for the relative intensity difference. The flow direction is top-down. The position of the initial laser tag is at a channel length of $0 \mathrm{~mm}$. The standard deviation of the relative intensity difference in the channel is 0.0047 , resulting in a signal-to-noise ratio on the order of 10 .

Figure 7 shows an equivalent result as Fig. 6 but with three averaged acquisitions. The standard deviation reduces to 0.0036 .

\section{E. Influence of methyl- $\beta$-cyclodextrin}

For examination of the influence of methyl $\beta-\mathrm{CD}$, its concentration is increased from $0-15$ $\mathrm{mmol} / \mathrm{L}$ in the initial CCVJ stock solution. Subsequently, images were taken with the same experimental parameters as in the preceding flow experiments.

Figure 8 shows a comparison with a concentration of $0 \mathrm{mmol} / \mathrm{L}$ (left) and $3 \mathrm{mmol} / \mathrm{L}$ (center) $120 \mathrm{~s}$ after tagging of ten averaged acquisitions. A Wiener filter with a window size of five pixels is applied. The laser tag is situated at a channel length of $0 \mathrm{~mm}$. The right subfigure represents the 


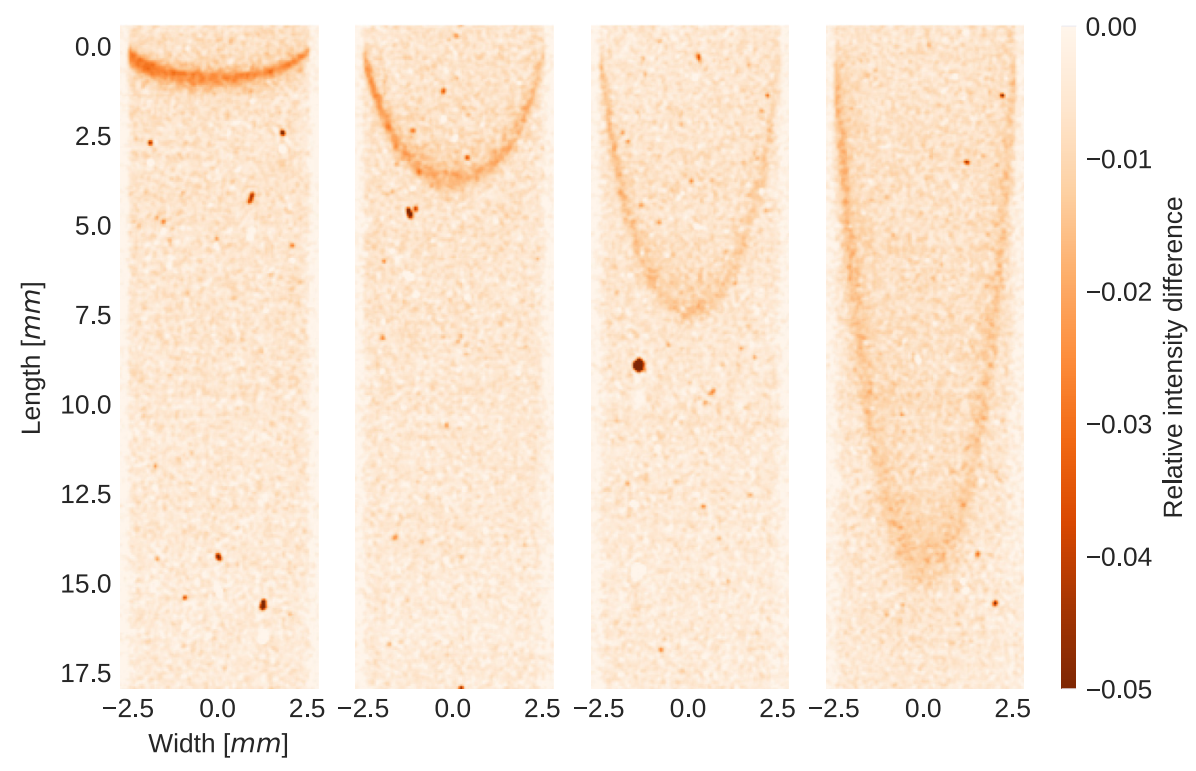

FIG. 7. Relative intensity difference with $\mathrm{Re}=9 \times 10^{-3}$ and $\mathrm{Pe}=1009$ at $10,50,100$, and $200 \mathrm{~s}$ (left to right) after a tag of $0.05 \mathrm{~s}$ for three averaged acquisitions. The standard deviation of the relative signal is 0.0036 .

difference in the results. Note that this subfigure has an increased noise level due to the two initial results' different computations. An increase due to methyl- $\beta$-CD is observable.

\section{F. Measurement with a long time difference}

In a third experiment, measurements of the flow up to 50-mm length of the channel were performed, which is the most extended feasible length in the setup.

In contrast to the preceding experiment of the Poiseuille flow, the laser activation time is increased to $4 \mathrm{~s}$. Furthermore, the solution contains $15 \mathrm{mmol} / \mathrm{L}$ methyl- $\beta$-CD.

Figure 9 shows the relative intensity differences of a single acquisition after 360, 460,600, and $720 \mathrm{~s}$. The laser tag is situated at a channel length of $0 \mathrm{~mm}$. A Wiener filter with a window size of five pixels is applied. The missing signal at a channel length of $12.5 \mathrm{~mm}$ on the left side is due to a scratch on the chamber surface. The standard deviation of the relative intensity difference in the channel is 0.0057 , resulting in a signal-to-noise ratio up to 10 .

In addition, Fig. 10 shows the results for the same experiment but with three averaged acquisitions. The standard deviation is 0.0047 .

\section{DISCUSSION}

\section{A. Comparison of the flow profile with theoretical Poiseuille flow}

In the low Re-number flow regime, the velocity profile can be approximated by an ideal laminar Poiseuille flow. Its analytical solution in a rectangular channel with width $L$ in the $y$ direction and depth $a$ in the $x$ direction is given by

$$
u(x, y)=\frac{1}{\mu} \frac{\partial p}{\partial z}\left[\frac{1}{2}\left(y^{2}-\frac{L^{2}}{4}\right)-4 L^{2} \sum_{n=1}^{\infty} \frac{(-1)^{n}}{s_{n}^{3}} \frac{\cosh \left(s_{n} K \frac{x}{a}\right)}{s_{n} K \frac{1}{2}} \cos \left(s_{n} \frac{y}{L}\right)\right],
$$




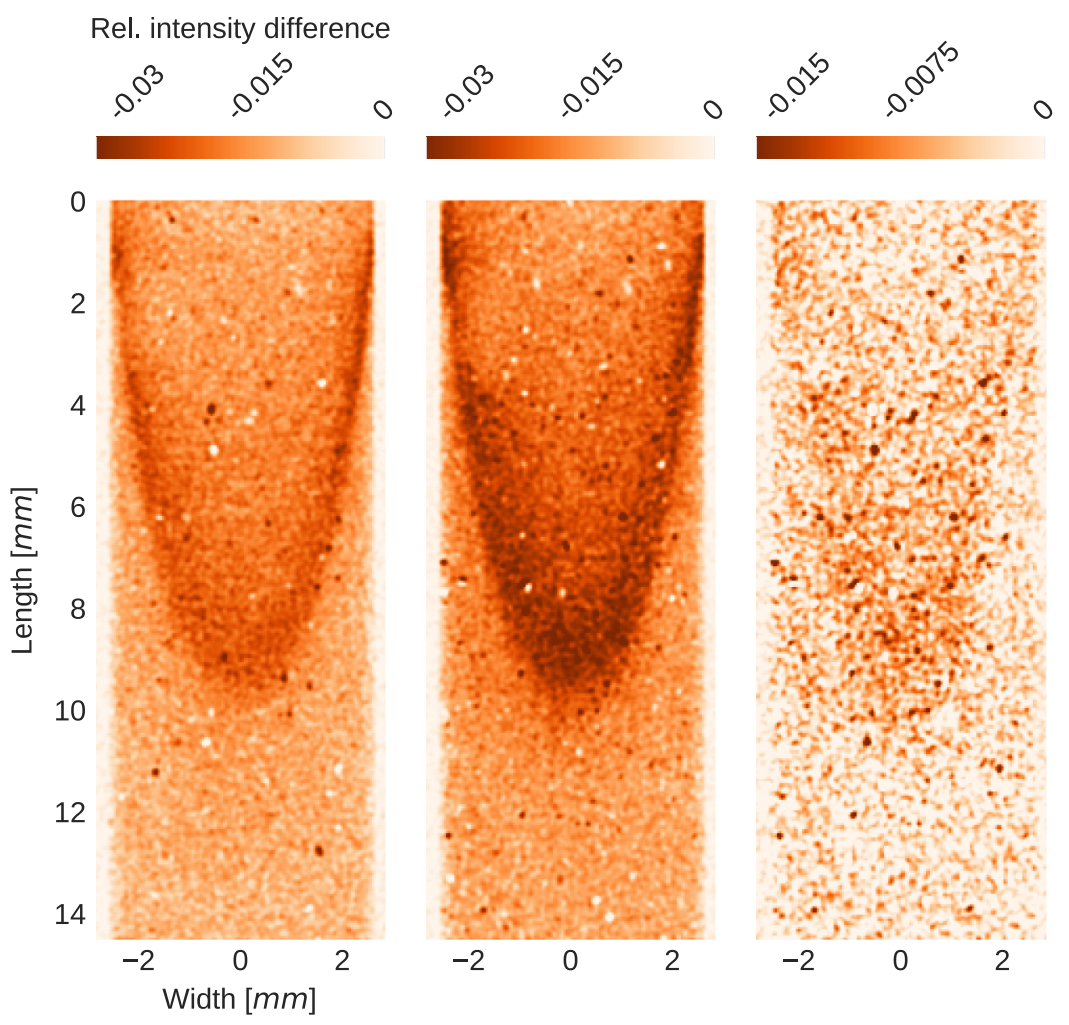

FIG. 8. Relative intensity difference with $\mathrm{Re}=9 \times 10^{-3}$ and $\mathrm{Pe}=1009$ at $120 \mathrm{~s}$, the concentration of methyl $-\beta$-CD of $0 \mathrm{mmol} / \mathrm{L}$ (left) and $3 \mathrm{mmol} / \mathrm{L}$ (center) for ten averaged acquisitions. The right subfigure is the difference of the two. Note the different color-bar limits of the right subfigure.

with $\partial p / \partial z$ as the pressure gradient in the flow direction, $s_{n}=(2 n-1) \pi$, and $K=a / L$ [43]. The origin of the coordinate system is in the center of the channel.

The mean flow velocity is calculated by double integration [43] and becomes

$$
\bar{u}=-\frac{1}{\mu} \frac{\partial p}{\partial z} \frac{L^{2}}{12}\left[1-\frac{192}{K} \sum_{n=1}^{\infty} \frac{1}{s_{n}^{5}} \tanh \left(s_{n} K \frac{1}{2}\right)\right] .
$$

Since $\bar{u}$ and the channel dimensions are given by the experiments, it is possible to compute the Poiseuille flow profile $u(x, y)$ by substitution of Eq. (7) in Eq. (6) and elimination of the pressure gradient term. A two-dimensional profile is computed at the center of the channel $(x=0)$ with the assumption of a tagging with only a small depth. For the approximation in Eq. (6) the summation is limited to $n=100$.

The results from Fig. 7 are further processed for comparison with the theoretical flow profile. The solution of the advection-diffusion equation for the presented problem with the concentration of the $E$ isomer as the observed parameter can be approximated with a Gaussian distribution in the streamwise direction,

$$
c_{E}(z, t)=-c_{E, t} \exp \left[-\frac{1}{2}\left(\frac{z-\mu_{m}(t)}{\sigma}\right)^{2}\right]+c_{E, \mathrm{amb}},
$$

with $c_{E, t}$ as the initial concentration after tagging, $\mu_{m}(t)$ as the expected mean value, $\sigma^{2}$ as the variance, and $c_{E \text {, amb }}$ representing the ambient concentration. For each column in the images, LMFIT 


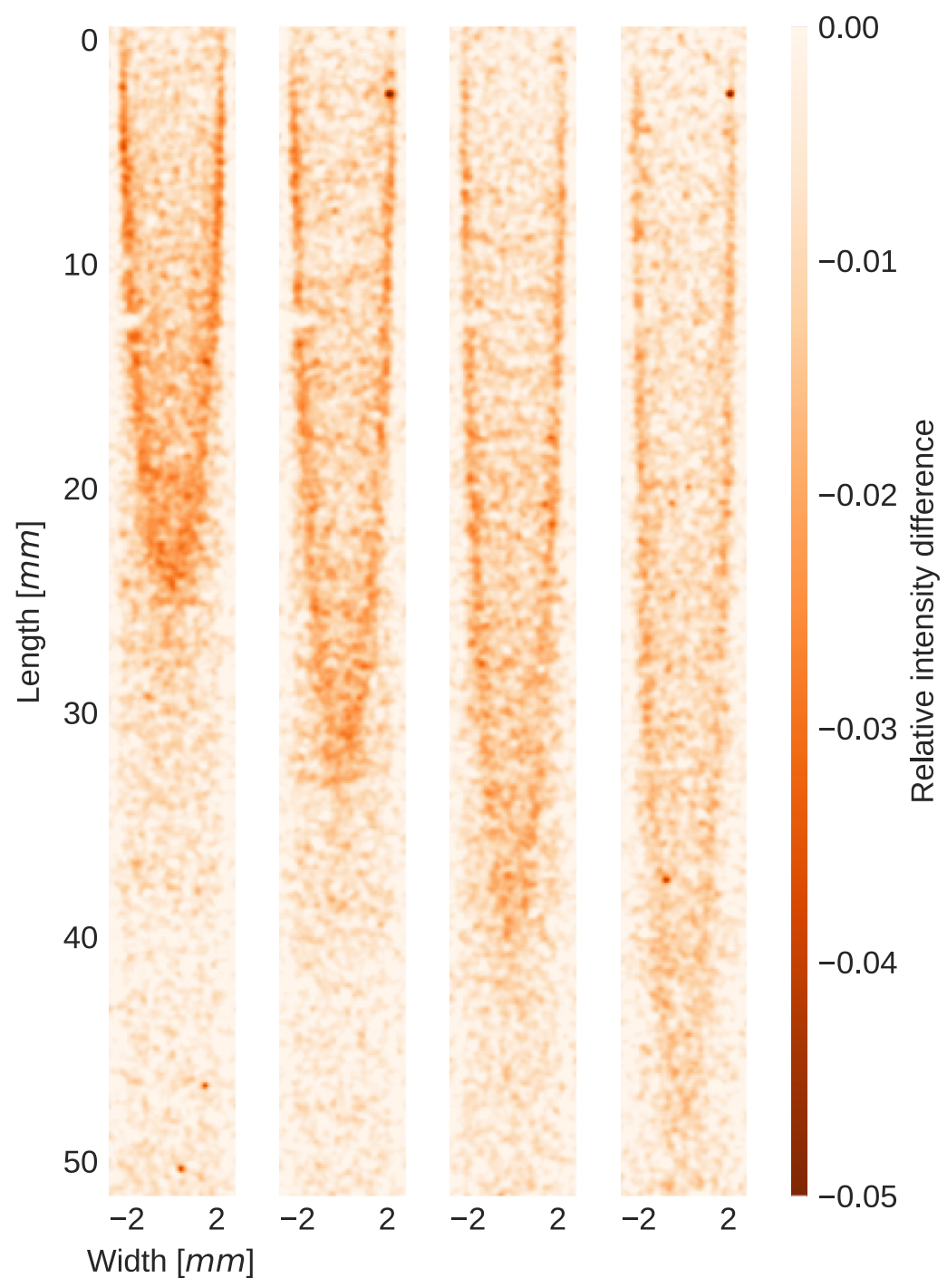

FIG. 9. Relative intensity difference with $\mathrm{Re}=9 \times 10^{-3}$ and $\mathrm{Pe}=1009$ at $360,460,600$, and $720 \mathrm{~s}$ (left to right) after a tag of $4 \mathrm{~s}$ for a single acquisition. The standard deviation of the relative signal is 0.0057 .

is used to compute a fit [40]. It is assumed that the concentration of the species is proportional to the intensity of the acquisition. The initial guess for the expected mean is computed with a median filter of 40 pixel size applied to the original image. The position guess is defined as the median of the 20 minimal values. The variance is computed by the difference of the 45 and 55 percentiles of each column. The initial reduced concentration is approximated with the difference in tagged and untagged areas. Figure 11 shows a comparison of the computed Poiseuille flow and the velocity of the tag in the experiment as in Fig. 7. The experimental results are in good agreement with the analytical solution. Note that the laser had a slight angle with regard to the channel wall, resulting in a slight offset in the computation of velocity.

\section{B. Influence of methyl- $\beta$-CD}

The intensity change of methyl- $\beta$-CD is detectable in the present setup. The results of Gavvala et al. [37] indicate a higher increase in fluorescence quantum yield than the results indicate. 


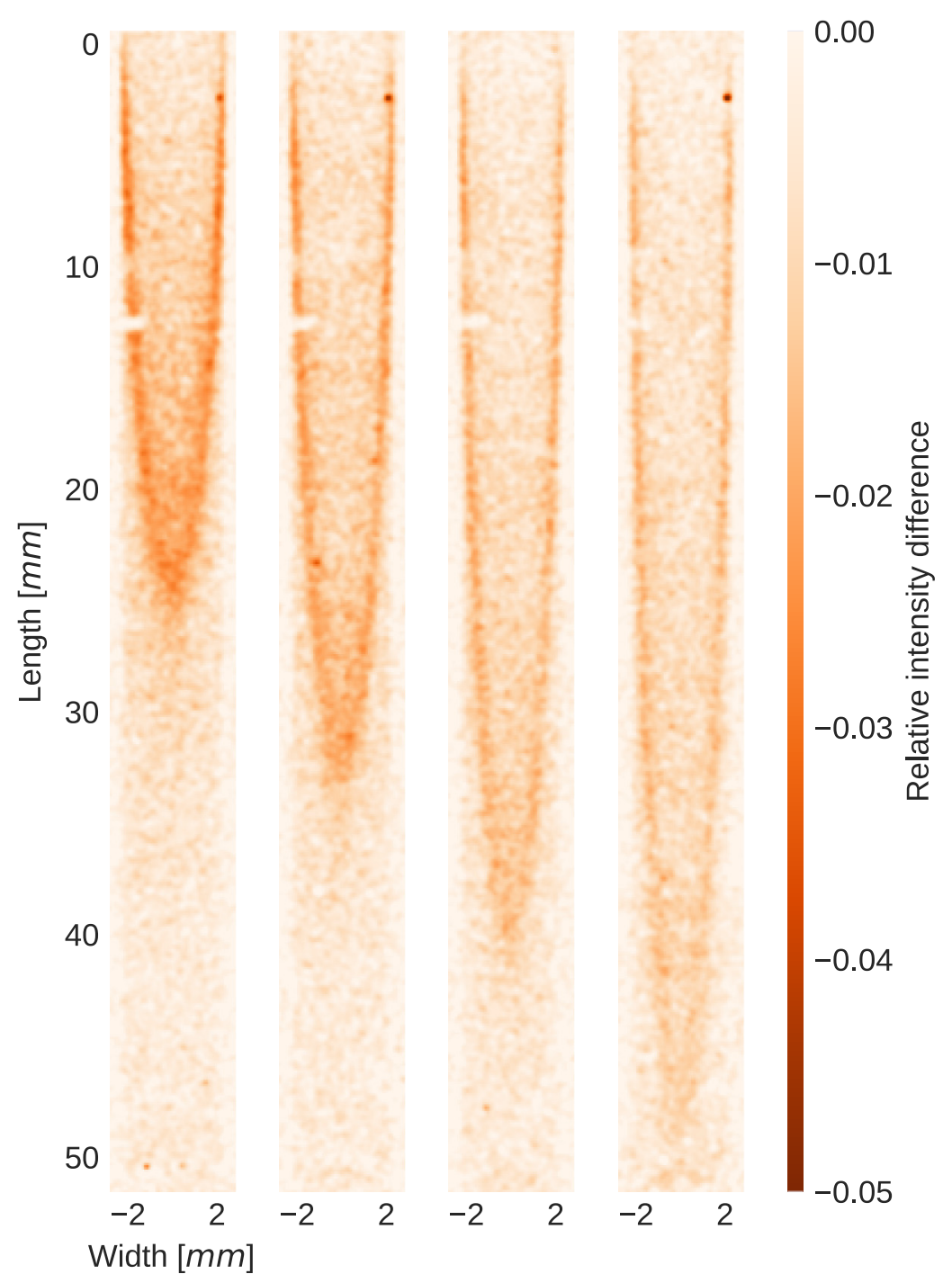

FIG. 10. Relative intensity difference with $\mathrm{Re}=9 \times 10^{-3}$ and $\mathrm{Pe}=1009$ at $360,460,600$, and $720 \mathrm{~s}$ (left to right) after a tag of $4 \mathrm{~s}$ for three averaged acquisitions. The standard deviation of the relative signal is 0.0047 .

Within the scope of photoisomeric MTV, there is another effect to be taken into account. Besides the increase in the fluorescence quantum yield due to the residence of the CCVJ molecule in the nanocavity, a decrease due to deexcitation by rotation of the functional group is anticipated. It is shown that the increase in fluorescence is higher than the decrease in photoisomerization. The applied CD has a carboxylic group as a lid on the complex. In the application of direct phosphorescence, it has been shown that the use of different lids, for example, alcoholic groups [44] or glycosyl subunits [45,46] have a strong influence on the luminescent behavior of the supramolecule. Further investigations of these complexes in combination with CCVJ appear desirable.

\section{CONCLUSION}

The photoisomeric dye CCVJ has been characterized from an experimental point of view. The recovery and tagging time constants have been experimentally determined as well as the diffusion 


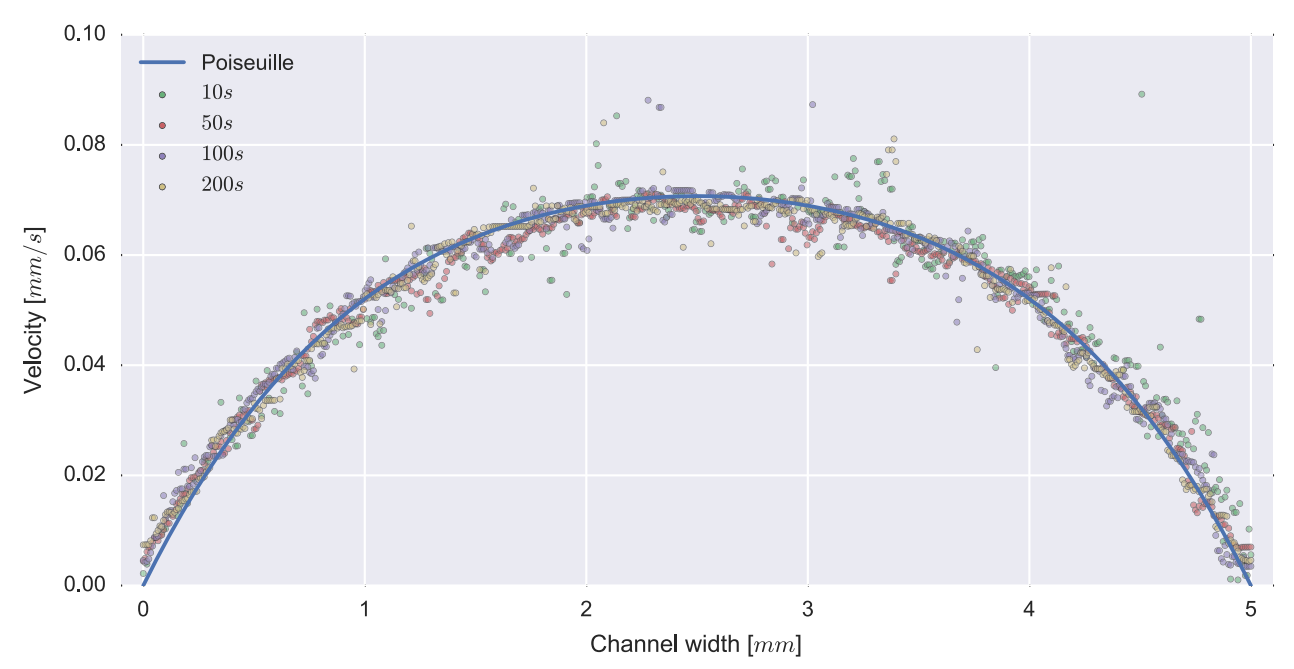

FIG. 11. Comparison of the analytical result of the Poiseuille flow with experimental data from Fig. 7. The single points are the result of a fit for each pixel column to Eq. (8) and the computation of the effective velocity. The results collapse to the Poiseuille profile for all observed times.

coefficient in ethylene glycol. The dye is suitable for long timescales between tagging and image acquisition due to its long recovery time constant. Furthermore, the simultaneous application of methyl- $\beta$-CD increases the recovery time further, enabling a higher contrast in the experiments. Photoisomeric MTV has been successfully applied to a Poiseuille flow at $\operatorname{Re}=9 \times 10^{-3}$ and $\mathrm{Pe}=$ 1009 , enabling computation of the velocities.

\section{A. Comparison with existing MTV methods}

In general, the presented method is cost-efficient in the setup. It could be demonstrated that it can measure liquid phase flows at low Re numbers. The temporal limits are defined by the isomerization rate constants of the chemical species.

In contrast to MTV by absorbance with photochromic dyes, the dynamic range can potentially be higher. Due to limited laser power available, this could not be investigated in the present experiment. A change in the tagging light source or observations on shorter timescales may further increase the achievable intensity change.

In comparison with MTV based on other photoproducts, such as caged dyes, the advantage is given by the regeneration of the isomer to its initial state. Furthermore, the dyes used are more cost efficient than caged dyes.

The difference with MTV by direct phosphorescence lies in the different timescales. The photoisomeric MTV may be applied in the same experiments as the phosphorescent supramolecules, but the expected dynamic range is low.

Overall, photoisomeric MTV offers a cost-efficient alternative with a simple setup to existing methods, especially when considering the regeneration ability in contrast to photocaged dyes.

\section{B. Comparison with other flow diagnostic methods}

In comparison with particle imaging and tracking methods, the application of photoisomeric MTV has similar distinctive advantages and disadvantages in terms of flow diagnostics as the existing applications of MTV. The use of the photoisomeric method might be advantageous in sensitive environments. Some measurements in environmental and biological fluid dynamics, especially in vivo for living organisms or in situ for ecosystems, do not permit the use of particles. 
One such example has been presented by Namykin et al. with the intravital measurement of a cerebral blood flow with Evans Blue [31]. The discussed molecular rotor CCVJ is already in use in biological applications, making it a suitable candidate for such use cases. Flows in porous media where the velocities are slow and tracer particles could block the microchannels are an example of environmental flows applicable to photoisomeric MTV.

\section{Fields of application}

The canonical laminar Poiseuille flow represents a slow flow regime. The tagging with the photoisomeric effect can, however, be applied in different scenarios. Concerning achievable timescales, the upper limit is virtually defined by the diffusion limit. Since the recovery time constant has been experimentally determined as $1050 \mathrm{~s}$, in most applications, the diffusion will deteriorate the tag before the isomerization decreases the intensity contrast. For turbulent flows, the lower limit is of relevance. With the used diode laser, the shortest possible tagging time constant is $26 \mathrm{~ms}$, which is not short enough to resolve turbulent timescales. As can be seen in Fig. 5, the tagging time constant is limited by the laser power density. The use of a higher power pulsed laser might decrease the required tagging time to timescales suitable for the acquisition of turbulent flows.

The fluid's viscosity has a significant influence on the method's performance, effectively altering the fluorescence lifetime as demonstrated by Rumble et al. [35]. For the neutral form of CCVJ, the lifetimes in methanol and ethylene glycol are 6 and 28 ps, respectively. Furthermore, the protonation state of $\mathrm{CCVJ}$ is of importance for the lifetimes. As a consequence, the lifetime depends significantly on the $p \mathrm{H}$ value of the solution [35]. To summarize, a fluid with a high viscosity, enabling the presence of CCVJ as an anion, is favorable for the presented method.

As demonstrated by Rumble et al., the achievable contrast can reach up to $20 \%$ between untagged and tagged samples [35]. This maximum contrast can only be achieved in a suitable setup. Rumble et al. [35] used a cuvette and tagged the solution completely. The tagging is achieved with a defined laser beam in the present setup, but the subsequent illumination is global. Hence, the observed signal always includes a region of untagged CCVJ, effectively decreasing the maximum achievable contrast. This effect has been demonstrated in Fig. 4 for the filter with a relative transmittance of 0.621 . The effectively tagged region in the cuvette was decreased, limiting the achievable contrast.

Data are available in a repository [42].

[1] B. Mckeon, G. Comte-Bellot, J. Foss, J. Westerweel, F. Scarano, and C. Tropea, Velocity, vorticity, and Mach number, in Springer Handbook of Experimental Fluid Mechanics, edited by C. Tropea, A. L. Yarin, and J. F. Foss (Springer, Berlin/Heidelberg, 2007), pp. 215-471.

[2] W. R. Lempert, Molecular tagging velocimetry, in Flow Visualization: Techniques and examples, edited by A. J. Smits (Imperial College Press, London, 2003), pp. 73-92.

[3] W. R. Lempert, P. Ronney, K. Magee, K. R. Gee, and R. P. Haugland, Flow tagging velocimetry in incompressible flow using photo-activated nonintrusive tracking of molecular motion (PHANTOMM), Exp. Fluids 18, 249 (1995).

[4] S. R. Harris, R. B. Miles, and W. R. Lempert, Observations of fluid flow produced in a closed cylinder by a rotating lid using the PHANTOMM (Photo-Activated Non Intrusive Tracking of Molecular Motion) flow tagging technique, in Eighth International Symposium on Applications of Laser Techniques to Fluid Mechanics, Lisbon, Portugal, 1996, edited by R. J. Adrian, D. F. G. Durao, F. Durst, M. V. Heitor, M. Maeda, and J. H. Whitelaw (Springer, Berlin, 1997), pp. 8-11.

[5] C. P. Gendrich, M. M. Koochesfahani, and D. G. Nocera, Molecular tagging velocimetry and other novel applications of a new phosphorescent supramolecule, Exp. Fluids 23, 361 (1997). 
[6] S. R. Harris, W. R. Lempert, L. Hersh, C. L. Burcham, D. A. Saville, R. B. Miles, K. Gee, and R. P. Haughland, Quantitative measurements of internal circulation in droplets using flow tagging velocimetry, AIAA J. 34, 449 (1996).

[7] M. Biage, S. R. Harris, W. R. Lempert, and A. J. Smits, Quantitative velocity measurements in turbulent Taylor-Couette flow by PHANTOMM flow tagging, in 8th International Symposium on Applications of Laser Techniques to Fluid Mechanics, Lisbon, Portugal, 1996, edited by R. J. Adrian, D. F. G. Durao, F. Durst, M. V. Heitor, M. Maeda, and J. H. Whitelaw (Springer, Berlin, 1997).

[8] P. H. Paul, M. G. Garguilo, and D. J. Rakestraw, Imaging of pressure- and electrokinetically driven flows through open capillaries, Anal. Chem. 70, 2459 (1998).

[9] J. S. Park and H. J. Kim, Molecular tagging fluorescence velocimetry (MTFV) for Lagrangian flow field mapping inside evaporating meniscus: Potential use for microscal applications, J. Flow Visualization Image Process. 8, 2 (2001).

[10] D. Sinton, X. Xuan, and D. Li, Thermally induced velocity gradients in electroosmotic microchannel flows: the cooling influence of optical infrastructure, Exp. Fluids 37, 872 (2004).

[11] J. Rička, Photobleaching velocimetry, Exp. Fluids 5, 381 (1987).

[12] S. Hosokawa, T. Fukunaga, and A. Tomiyama, Application of photobleaching molecular tagging velocimetry to turbulent bubbly flow in a square duct, Exp. Fluids 47, 745 (2009).

[13] K. F. Schrum, J. M. Lancaster, S. E. Johnston, and S. D. Gilman, Monitoring Electroosmotic Flow by Periodic Photobleaching of a Dilute, Neutral Fluorophore, Anal. Chem. 72, 4317 (2000).

[14] B. Mosier, J. Molho, and J. Santiago, Photobleached-fluorescence imaging of microflows, Exp. Fluids 33, 545 (2002).

[15] F. Brunet, E. Cid, A. Bartoli, E. Bouche, F. Risso, and V. Roig, Image registration algorithm for molecular tagging velocimetry applied to unsteady flow in Hele-Shaw cell, Exp. Therm. Fluid Sci. 44, 897 (2013).

[16] K. Roetmann, W. Schmunk, C. S. Garbe, and V. Beushausen, Micro-flow analysis by molecular tagging velocimetry and planar Raman-scattering, Exp. Fluids 44, 419 (2008).

[17] B. Stier and M. M. Koochesfahani, Molecular Tagging Velocimetry (MTV) measurements in gas phase flows, Exp. Fluids 26, 297 (1999).

[18] R. W. Pitz, M. D. Lahr, Z. W. Douglas, J. A. Wehrmeyer, S. Hu, C. D. Carter, K.-Y. Hsu, C. Lum, and M. M. Koochesfahani, Hydroxyl tagging velocimetry in a supersonic flow over a cavity, Appl. Opt. 44, 6692 (2005).

[19] M. A. Mustafa, D. Shekhtman, and N. J. Parziale, Single-Laser Krypton Tagging Velocimetry Investigation of Supersonic Air and $\mathrm{N}_{2}$ Boundary-Layer Flows over a Hollow Cylinder in a Shock Tube, Phys. Rev. Appl. 11, 064013 (2019).

[20] N. J. Parziale, M. S. Smith, and E. C. Marineau, Krypton tagging velocimetry of an underexpanded jet, Appl. Opt. 54, 5094 (2015).

[21] D. Zahradka, N. J. Parziale, M. S. Smith, and E. C. Marineau, Krypton tagging velocimetry in a turbulent Mach 2.7 boundary layer, Exp. Fluids 57, 62 (2016).

[22] M. A. Mustafa and N. J. Parziale, Simplified read schemes for krypton tagging velocimetry in $\mathrm{N}_{2}$ and air, Opt. Lett. 43, 2909 (2018).

[23] W. R. Lempert, N. Jiang, S. Sethuram, and M. Samimy, Molecular tagging velocimetry measurements in supersonic microjets, AIAA J. 40, 1065 (2002).

[24] J. B. Michael, M. R. Edwards, A. Dogariu, and R. B. Miles, Femtosecond laser electronic excitation tagging for quantitative velocity imaging in air, Appl. Opt. 50, 5158 (2011).

[25] N. Jiang, B. R. Halls, H. U. Stauffer, P. M. Danehy, J. R. Gord, and S. Roy, Selective two-photon absorptive resonance femtosecond-laser electronic-excitation tagging velocimetry, Opt. Lett. 41, 2225 (2016).

[26] R. A. Burns, P. M. Danehy, B. R. Halls, and N. Jiang, Femtosecond laser electronic excitation tagging velocimetry in a transonic, cryogenic wind tunnel, AIAA J. 55, 680 (2017).

[27] R. Sánchez-González, R. Srinivasan, R. D. W. Bowersox, and S. W. North, Simultaneous velocity and temperature measurements in gaseous flow fields using the VENOM technique, Opt. Lett. 36, 196 (2011). 
[28] R. Sánchez-González, R. D. W. Bowersox, and S. W. North, Simultaneous velocity and temperature measurements in gaseous flowfields using the vibrationally excited nitric oxide monitoring technique: a comprehensive study, Appl. Opt. 51, 1216 (2012).

[29] F. Pan, R. Sánchez-González, M. H. McIlvoy, R. D. W. Bowersox, and S. W. North, Simultaneous threedimensional velocimetry and thermometry in gaseous flows using the stereoscopic vibrationally excited nitric oxide monitoring technique, Opt. Lett. 41, 1376 (2016).

[30] A. A. Namykin, A. P. Khorovodov, O. V. Semyachkina-Glushkovskaya, V. V. Tuchin, and I. V. Fedosov, Photoinduced enhancement of evans blue dye fluorescence in water solution of albumin, Opt. Spectrosc. 126, 554 (2019).

[31] A. A. Namykin, N. A. Shushunova, M. V. Ulanova, O. V. Semyachkina-Glushkovskaya, V. V. Tuchin, and I. V. Fedosov, Intravital molecular tagging velocimetry of cerebral blood flow using Evans Blue, J. Biophotonics 11, e201700343 (2018).

[32] M. A. Haidekker, W. Akers, D. Lichlyter, T. P. Brady, and E. A. Theodorakis, sensing of flow and shear stress using fluorescent molecular rotors, Sens. Lett. 3, 42 (2005).

[33] A. Mustafic, H.-M. Huang, E. A. Theodorakis, and M. A. Haidekker, Imaging of flow patterns with fluorescent molecular rotors, J. Fluoresc. 20, 1087 (2010).

[34] A. Mustafic, K. M. Elbel, E. A. Theodorakis, and M. Haidekker, Apparent shear sensitivity of molecular rotors in various solvents, J. Fluoresc. 25, 729 (2015).

[35] C. Rumble, K. Rich, G. He, and M. Maroncelli, CCVJ is not a simple rotor probe, J. Phys. Chem. A 116, 10786 (2012).

[36] M. J. Schmidt, D. Sauter, and T. Rösgen, Flow-dependent fluorescence of CCVJ, J. Biol. Eng. 11, 24 (2017).

[37] K. Gavvala, S. Satpathi, and P. Hazra, Ultrafast dynamics of a molecular rotor in chemical and biological nano-cavities, RSC Adv. 5, 72793 (2015).

[38] M. J. Schmidt, B. Kaeslin, and T. Rösgen, Molecular tagging velocimetry using photoisomerization, in Proceedings 18th International Symposium on Flow Visualization, edited by T. Rösgen (ETH Zurich, Zurich, Switzerland, 2018).

[39] J. Saltiel and Y. P. Sun, Cis-trans isomerization of $\mathrm{C}=\mathrm{C}$ double bonds, in Photochromism: Molecules and System (Elsevier Science, Amsterdam, 2003), pp. 64-164.

[40] M. Newville, T. Stensitzki, D. B. Allen, and A. Ingargiola, LMFIT: Non-Linear Least-Square Minimization and Curve-Fitting for Python, Zenodo.org (CERN, Meyrin, Switzerland, 2014).

[41] R. M. Wellek, R. D. Mitchell, and J. W. Moore, Diffusion coefficients of ethylene glycol and cyclohexanol in the solvents ethylene glycol, diethylene glycol, and propylene glycol as a function of temperature, J. Chem. Eng. Data 16, 57 (1971).

[42] M. J. Schmidt, B. Käslin, and T. Rösgen, Photoisomeric MTV data for Poiseuille flow with CCVJ, doi: 10.5281/zenodo.4412146 (2020).

[43] W. Rybiński and J. Mikielewicz, Analytical solutions of heat transfer for laminar flow in rectangular channels, Arch. Thermodyn. 35, 29 (2015).

[44] W. K. Hartmann, M. H. B. Gray, A. Ponce, D. G. Nocera, and P. A. Wong, Substrate induced phosphorescence from cyclodextrin-lumophore host-guest complexes, Inorganica Chimica Acta 243, 239 (1996).

[45] H. Hu, M. Koochesfahani, and C. Lum, Molecular tagging thermometry with adjustable temperature sensitivity, Exp. Fluids 40, 753 (2006).

[46] $\mathrm{H}$. Hu and M. M. Koochesfahani, Molecular tagging velocimetry and thermometry and its application to the wake of a heated circular cylinder, Meas. Sci. Technol. 17, 1269 (2006). 